\title{
Utility of Ultrasound in the Diagnosis, Treatment, and Follow-up of Prostate Cancer: State of the Art
}

\author{
Frank K. Chen ${ }^{1}$, Andre Luis de Castro Abreu ${ }^{2}$, and Suzanne L. Palmer ${ }^{1}$ \\ ${ }^{I}$ Department of Radiology, Keck Medical Center of USC, Los Angeles, California; and ${ }^{2}$ Department of Urology, Keck Medical Center \\ of USC, Los Angeles, California
}

\begin{abstract}
Prostate cancer screening currently consists of serum prostatespecific antigen and digital rectal examination, followed by transrectal ultrasound-guided biopsy for diagnostic confirmation. Although the current paradigm of prostate cancer screening has led to a decrease in advanced disease and cancer-related mortality, these techniques have limitations in terms of sensitivity and specificity, resulting in missed cancers that are clinically significant and the overdetection of clinically insignificant cancers. New imaging techniques and technologies are required to improve the detection of prostate cancer. This article summarizes the use of novel ultrasound techniques and technologies in the detection, biopsy, and treatment of prostate cancer.
\end{abstract}

Key Words: genitourinary; Doppler ultrasound; MRI/TRUS targeted biopsy; prostate cancer; surveillance biopsy; transrectal ultrasound

J Nucl Med 2016; 57:13S-18S

DOI: 10.2967/jnumed.116.177196

\section{$\mathbf{P}$} with approximately 180,000 new cases diagnosed and 26,000 cancerrelated deaths projected in the United States in 2016 (1). With the introduction of prostate-specific antigen (PSA) screening in the late 1980s, the known incidence of prostate cancer has increased substantially because of earlier detection in asymptomatic men, peaking in 1992 (2). The risk factors for developing prostate cancer include age, ethnicity, genetics, and dietary factors. Prostate cancer is a disease of older men, rarely diagnosed before the age of 50 years, with the incidence increasing exponentially after that age (3). African-Americans have a 1.5 times greater incidence of prostate cancer than whites and a more advanced stage of disease at the time of diagnosis (4).

Many factors affect prostate cancer survival, especially the disease extent at the time of diagnosis. Early detection and treatment are vital if a cure or improved patient survival is desired. Currently, serum PSA and digital rectal examination are used for screening. If either study is abnormal, transrectal ultrasound (TRUS)-guided biopsy is performed for diagnostic confirmation. Although the current paradigm of prostate cancer screening has led to a decrease in advanced disease and cancer-related mortality, these techniques

Received Apr. 22, 2016; revision accepted Aug. 8, 2016.

For correspondence or reprints contact: Frank K. Chen, Department of Radiology, Keck Medical Center of USC, 1500 San Pablo St., Los Angeles, CA 90033.

E-mail: frank.chen@med.usc.edu

COPYRIGHT (C 2016 by the Society of Nuclear Medicine and Molecular Imaging, Inc. have limitations in terms of sensitivity and specificity, resulting in missed cancers that are clinically significant and the overdetection of clinically insignificant cancers. Overdetection leads to the overtreatment of prostate cancer and in the process exposes patients to unnecessary side effects related to treatment (5-7). A clinically significant cancer has been defined as a tumor with a volume greater than $0.5 \mathrm{~cm}^{3}$ and a Gleason score of 7 or greater.

Because of the limitations in current screening tests, new techniques and technologies are needed to improve the detection of clinically significant prostate cancer while at the same time limiting the overdetection of clinically insignificant cancer. In this article, we review the use of new ultrasound techniques, including contrastenhanced ultrasound (CEUS) and ultrasound elastography, in the imaging of prostate cancer. We also review the role of ultrasound in the biopsy and locoregional treatment of prostate cancer.

\section{GRAY-SCALE ULTRASOUND}

Gray-scale TRUS, a cost-effective and readily available imaging modality, is the most commonly used radiologic study for the evaluation of the prostate gland. The use of TRUS in the detection of prostate cancer has limited diagnostic accuracy. Most prostate cancers $(60 \%-80 \%)$ are hypoechoic on TRUS, whereas $30 \%-40 \%$ of prostate cancers are isoechoic, and approximately $1.5 \%$ are hyperechoic (Fig. 1) (8). Among ultrasound-evident lesions, only $17 \%-57 \%$ are malignant. Benign entities, such as prostatitis, atrophy, infarction, and benign prostatic hypertrophy, may be hypoechoic on TRUS. The sensitivity and specificity of TRUS are limited, ranging between $40 \%$ and $50 \%$ for detecting prostate cancer. Lesions on TRUS are better visualized in the peripheral zone than in the transitional zone because of the heterogeneous pattern of the latter (9). Additional findings on TRUS that may suggest, but are not specific for, underlying malignancy include bulging or irregularity of the prostatic capsule and asymmetry of the prostate gland in terms of morphology and echogenicity (10).

The use of TRUS for local staging of prostate cancer is inadequate. The diagnostic accuracy of TRUS to evaluate extracapsular extension ranges from $37 \%$ to $85 \%$ and depends on clinician experience in performing and interpreting the study. TRUS findings that suggest extracapsular extension include capsular bulge or irregularity adjacent to a visible lesion and hypoechoic periprostatic fat stranding (11). Increased tumor contact (length $>23 \mathrm{~mm}$ ) with the prostatic capsule is associated with a higher probability of extracapsular extension (12).

\section{COLOR AND POWER DOPPLER ULTRASOUND}

The principle behind the use of color Doppler ultrasound (CDUS) and power Doppler ultrasound (PDUS) in the evaluation 


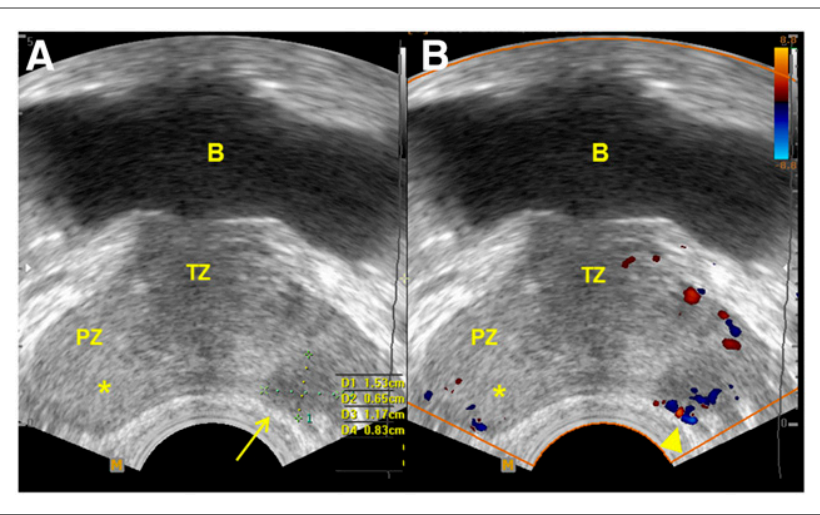

FIGURE 1. Hypoechoic and isoechoic prostate cancer on TRUS in 71 -y-old man with elevated PSA of $4.28 \mathrm{ng} / \mathrm{mL}$ and palpable nodule at prostate base. (A) Gray-scale image at prostate base demonstrates hypoechoic lesion with subtle left capsular bulging (arrow). (B) CDUS demonstrates increased blood flow within lesion (arrowhead). Biopsies directed into this lesion showed Gleason scores of 7-9. Biopsy directed into contralateral side (asterisk indicates no obvious lesion on TRUS) showed Gleason score of 7. $\mathrm{B}=$ urinary bladder; $\mathrm{TZ}=$ transitional zone; $\mathrm{PZ}=$ peripheral zone.

of prostate cancer is the detection of increased perfusion compared with surrounding prostate tissue related to tumor neovascularity. Three different flow patterns-diffuse flow within, focal flow within, and surrounding flow-have been found to be associated with prostate cancer on CDUS, with diffuse flow being the most common pattern. The combination of CDUS and gray-scale ultrasound has been shown to detect a greater number of prostate cancers than gray-scale ultrasound alone, with higher specificity and positive predictive value $(13,14)$. CDUS is especially useful in detecting isoechoic prostate cancers that demonstrate increased vascularity. Despite improved prostate cancer detection, CDUS has limited diagnostic accuracy because a substantial number of cancers are still missed, and it has relatively low specificity because not all tumors are hypervascular and not all hypervascular lesions are malignant $(15,16)$.

PDUS is more sensitive than CDUS in detecting flow, especially slow flow (Figs. 2A-2C). However, PDUS has not been shown to have increased benefit over CDUS for detecting prostate cancer. Studies suggest that on CDUS and PDUS, hypervascular and hypovascular lesions are associated with higher and lower Gleason scores, respectively (Fig. 1B) $(17,18)$.

\section{CEUS}

CEUS is an ultrasound technique that uses intravenously injected gas-filled microbubbles as a contrast agent to provide microvascular and tissue perfusion information. The microbubbles are encapsulated in a lipid or surfactant shell and have a diameter of $1-10 \mu \mathrm{m}$, which is smaller than the size of a red blood cell, enabling them to penetrate into capillary beds. CEUS is better at evaluating microvascularity than CDUS, which is limited by its ability to detect flow only in vessels larger than $1 \mathrm{~mm}$ in diameter. Unlike CT or MRI contrast, ultrasound contrast is an ideal agent for evaluating perfusion because it is a pure blood pool agent and does not diffuse into the surrounding tissue, which would obscure the microvasculature (19). Using various ultrasound techniques, most commonly pulse inversion or harmonic imaging, and using a low mechanical index to avoid destroying bubbles in a single pulse and thus allow longer periods of interrogation, nonlinear reflectors such as microbubbles are preferentially detected by ultrasound over linear reflectors such as normal tissue, allowing evaluation of the vasculature $(20)$.

The growth of prostate cancer induces neovascularization; as a result, prostate cancer is associated with increased microvessel density (21), which has been shown to be a prognostic marker of prostate cancer $(22,23)$. The role of CEUS in prostate cancer
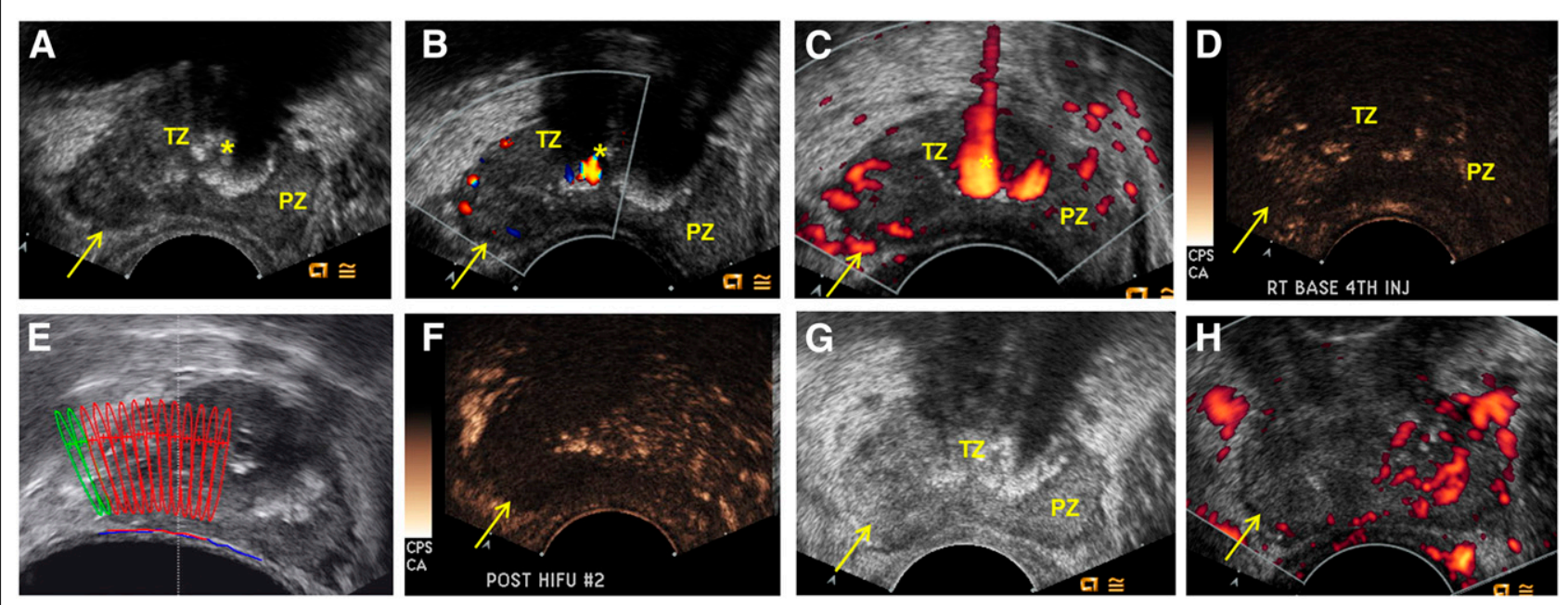

FIGURE 2. 63-y-old man with palpable nodule at right base and elevated PSA of $4.2 \mathrm{ng} / \mathrm{mL}$. (A-C) Gray-scale (A), CDUS (B), and PDUS (C) images obtained before HIFU. Gray-scale image demonstrates hypoechoic area at right base corresponding to palpable nodule (arrow). Asymmetric blood flow is seen on both CDUS and PDUS (arrows). PDUS is more sensitive to slow flow. (D) CEUS image before HIFU demonstrates early enhancement of lesion (arrow). (E) Image during HIFU demonstrates treatment plan. Areas in red have been treated; those in green have not been treated. (F) CEUS image after HIFU demonstrates no enhancement in treated area (arrow). (G and H) After HIFU, gray-scale (G) and PDUS (H) images demonstrate enlargement of, increased heterogeneity of, and decreased blood flow to treated area (arrow). Asterisk indicates calcification. TZ $=$ transitional zone; $\mathrm{PZ}=$ peripheral zone. 
imaging is to detect these microvessels. Sedelaar et al. (24) found that prostate cancer with increased microvessel density enhanced more on CEUS than did the corresponding normal contralateral side. On CEUS, findings suggestive of prostate cancer include focal rapid increased contrast enhancement and asymmetric prostate vascularity (Figs. 2D and 2F) (25). Studies have shown that CEUS is better than gray-scale ultrasound for detecting prostate cancer (26-28). In a metaanalysis that included 16 studies and 2,624 patients, Li et al. (29) found that CEUS had a pooled sensitivity and specificity of $70 \%$ and $74 \%$, respectively, for the detection of prostate cancer.

CEUS provides not only qualitative information but also quantitative information. Time-intensity curves can be generated in regard to a region of interest, which can be used to quantify enhancement parameters such as bolus arrival time, time to peak intensity, area under the curve, and wash-in and washout curves (30). Goossen et al. (31) found that time to peak intensity was the enhancement parameter with the greatest diagnostic accuracy, detecting $78 \%$ of cancers. Zhu et al. (32) found that higher-grade prostate cancers had a shorter bolus arrival time and time to peak intensity and a higher overall peak intensity.

\section{ULTRASOUND ELASTOGRAPHY}

Most prostate cancers are known to be harder and less elastic than normal prostate tissue, which allows them to be detected on digital rectal examination. This stiffening is secondary to increased cellular density, decreased glandular tissue architecture, and increased collagen deposition in the stroma surrounding the tumor (33). The basis of ultrasound elastography is the detection of the elastic difference between prostate cancers and surrounding prostatic tissue. The two types of ultrasound elastography are strain, or quasistatic, elastography (SE) and ShearWave (Aixplorer) Elastography (SWE; Aixplorer).

In SE, cyclical mechanical compressions and decompressions of the prostate gland are performed using a TRUS probe. An inflated balloon filled with water may be placed between the probe and the rectal wall to achieve more uniform compression. The difference in tissue displacement from one frame to another is used to generate an elastogram, which is a color map of local tissue strain (34). In a metaanalysis by Aboumarzouk et al. (35), the sensitivity and specificity of SE were in the range of $71 \%-82 \%$ and $60 \%-95 \%$, respectively, with radical prostatectomy specimens as the reference standard. A more recent metaanalysis by Zhang et al. (36) comparing SE with radical prostatectomy specimens reported a pooled sensitivity of $72 \%$ and specificity of $76 \%$. Benign conditions such as prostatitis, fibrosis, and atrophy may demonstrate decreased elasticity, resulting in false-positive results. The diagnostic accuracy of SE in detecting prostate cancer has been shown to be proportional to both tumor size and Gleason grade (37). SE is considerably operator-dependent because of subjectivity related to color map interpretation and difference in degree of free-hand cyclic compression between users. The quantification of tissue elasticity is not possible with SE because the color map is automatically scaled to the softest and stiffest regions in the imaged field (38).

Unlike SE, SWE does not require manual compression and decompression of the prostate gland, thus eliminating operator dependency. In SWE, an acoustic radiation force is generated by a focused ultrasound beam, resulting in a shear wave that propagates through the interrogated tissue. Stiffness can be measured on the basis of the velocity at which the waves propagate through the tissue, with faster velocities through harder tissue (39). The SWE (in $\mathrm{m} / \mathrm{s}$ ) can be used to calculate Young's modulus (in $\mathrm{kPa}$ ), a measure of tissue stiffness, and can then be expressed as a quantitative elasticity color map. SWE is a relatively novel technique with a limited amount of data available for it. Correas et al. (40), in a study of 184 men correlating with 12-core sextant biopsy, found a sensitivity, specificity, positive predictive value, and negative predictive value of $96 \%, 85 \%, 48 \%$, and $99 \%$, respectively, using a cutoff of $35 \mathrm{kPa}$. Using a threshold of $50 \mathrm{kPa}$ and correlating with whole-mount sections, Boehm et al. (41) reported a sensitivity, specificity, positive predictive value, and negative predictive value of $81 \%, 69 \%, 67 \%$, and $82 \%$, respectively, in a study of 60 patients. In a study correlating SWE with 12-core biopsy and SWE-guided targeted biopsy in 50 men, Ahmad et al. (42) reported a sensitivity and specificity of $90 \%$ and $88 \%$, respectively, in patients with a PSA of less than $20 \mu \mathrm{g} / \mathrm{L}$ and a sensitivity and specificity of $93 \%$ and $93 \%$, respectively, in patients with a PSA greater than $20 \mu \mathrm{g} / \mathrm{L}$. Those authors also found a relationship between Young's modulus and increasing Gleason score. Unlike other studies, the estimated stiffness values of malignant tissue were significantly greater than those of benign tissue (134 kPa vs. $75 \mathrm{kPa}$, respectively). Contrary to other published data, in a recent study of 73 patients by Porsch et al. (43) that correlated with 12-core biopsy, SWE was found to be a poor predictor of malignancy, regardless of Young's modulus cutoff. However, it was fairly reliable in distinguishing between lesions with a Gleason score of 6 or less and lesions with a Gleason score greater than 6 using a cutoff of $80 \mathrm{kPa}$.

Ultrasound elastography is limited in its evaluation of prostate cancers in the central and transitional zones and anterior prostate because of the increased heterogeneity and hardness in these regions and resultant signal attenuation.

\section{THE ROLE OF ULTRASOUND IN PROSTATE BIOPSY}

Prostate biopsy is usually indicated when one or more of the following factors exist: elevated PSA, rising PSA, abnormal findings on digital rectal examination, and prior biopsies demonstrating atypical small acinar neoplasia. Because of the low sensitivity and specificity of TRUS for the detection of prostate cancer, TRUS-guided targeted biopsy has limited value. However, because of its ability to clearly delineate prostate zonal anatomy, its ease of use, and its real-time capabilities, TRUS is the most commonly used modality for guiding systematic prostate biopsy. In 1989, Hodge et al. (44) introduced the use of TRUS to guide sextant biopsy of the prostate gland, which involves sampling of the parasagittal apex, midzone, and base of the right and left sides of the prostate gland. However, the sextant biopsy strategy has since been superseded by extended 10- to 12-core biopsy protocols, which involve performing the standard sextant biopsy plus additional biopsies of the far lateral and apical zones. Extended 10- to 12-core biopsy protocols increase cancer detection rates up to $30 \%$, increase negative predictive value, have a more accurate tumor grade concordance with radical prostatectomy, and do not increase the likelihood of detecting insignificant cancers (45). However, increasing the biopsy cores to more than 12 samples has not been shown to significantly increase cancer detection rates or negative predictive value but may increase the detection of insignificant cancers (46).

Several studies have compared CEUS- and ultrasound elastographytargeted biopsies with systematic biopsy protocols. Initial studies were 

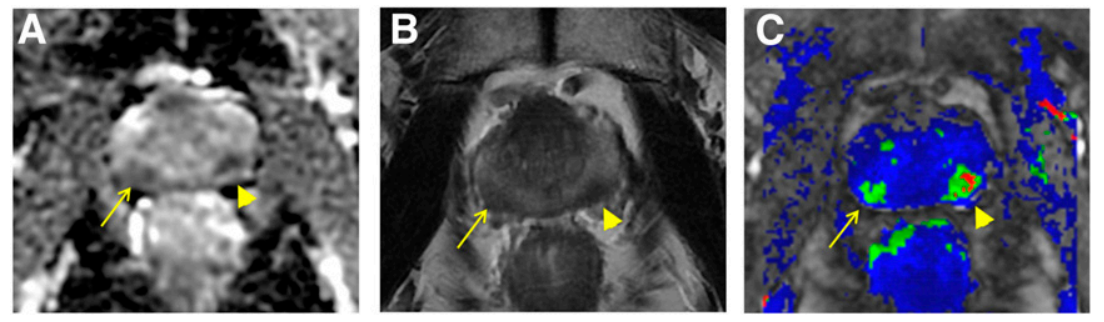

Cryoablation is a thermoablation technique that achieves cellular destruction by rapid cycles of freezing and thawing, resulting in coagulative necrosis. Under TRUS guidance, argon-based probes are advanced into the prostate gland via a transperineal approach. Pressurized argon gas is used to freeze the probe tip, which can reach temperatures as low as $-187^{\circ} \mathrm{C}$. The probe tip can then be rapidly thawed

FIGURE 3. Same 71-y-old man as in Figure 1. Within peripheral zone, prebiopsy multiparametric MRI demonstrates areas of restricted diffusion on apparent diffusion coefficient map (A), areas of decreased signal intensity on T2-weighted image (B), and areas of increased perfusion on dynamic contrast-enhanced image (C). MRI/TRUS coregistered targeted biopsy was performed. Lesion on left (arrowhead) was seen on TRUS; targeted biopsy showed Gleason score of 7-9. Lesion on right (arrow) was not seen on TRUS; targeted biopsy showed Gleason score of 7 .

by exchanging pressurized helium into the probe, rapidly reheating the tip to $67^{\circ} \mathrm{C}$. Achieving complete cell destruction requires temperatures lower than $-40^{\circ} \mathrm{C}(50)$. In a literature review, Marien et al. (51) reported that focal therapy of prostate cancer with cryoablation had a biopsy-free recur-

promising, some of which suggested that targeted biopsies using these novel ultrasound techniques had superior cancer detection rates and required fewer than half the number of cores required by systematic biopsy protocols. However, in a systematic literature review by van Hove et al. (47), no clear conclusion can be reached about the superiority or inferiority of ultrasound-targeted biopsies compared with systematic biopsies because of the large number of contradictory studies. However, in almost all studies, targeted biopsies combined with systematic biopsies had greater cancer detection rates, sometimes substantially greater, than systematic biopsies alone. Absolute cancer detection rates increased $2 \%-8 \%$ and $7 \%-15 \%$, respectively, when CEUS- and ultrasound elastography-targeted biopsies were combined with systematic biopsies.

MRI-ultrasound fusion is another novel imaging technique that is being used to guide prostate biopsy. The sensitivity and specificity of multiparametric MRI for the detection of prostate cancer are $88 \%$ and $74 \%$, respectively (48). Despite the relatively high sensitivity and specificity of multiparametric MRI, MRI-guided biopsy of the prostate is not widely used because of its limitations of time and cost. MRI-ultrasound fusion resolves this issue by combining the diagnostic advantages of MRI with the cost-effectiveness and ease of use of ultrasound. In MRI-ultrasound fusion biopsy of the prostate gland, multiparametric MRI of the prostate is obtained before the biopsy (Fig. 3). The MR images are then coregistered with real-time TRUS either cognitively or using commercially marketed MRI-ultrasound fusion platforms. Systematic biopsies and targeted biopsies of MRI-suspected lesions are performed. MRI-ultrasound fusion-guided biopsy has been shown to detect more clinically significant prostate cancers than systematic biopsies alone. In a metaanalysis that included 15 studies and 2,293 patients, Valerio et al. (49) found that, using fewer cores (median, 9.2 vs. 37.1), MRI-ultrasound fusion-guided biopsy detected more clinically significant cancers (median, $33 \%$ vs. $24 \%$ ) than systematic biopsy did.

\section{THE ROLE OF ULTRASOUND IN THE TREATMENT OF PROSTATE CANCER}

Traditional treatment options for clinically localized prostate cancer include radical prostatectomy, external beam radiation, brachytherapy, and active surveillance (Fig. 4). Two additional treatment options for clinically localized prostate cancer are cryoablation and high-intensity focused ultrasound (HIFU). rence rate of $60 \%-94 \%$. Posttreatment complications included erectile dysfunction $(15 \%-40 \%)$ and incontinence $(1 \%-10 \%)$.

In HIFU, high-intensity ultrasonic energy is focused on a fixed target. The deposition of a large amount of energy in a focused area results in cellular disruption and coagulative necrosis via two mechanisms, thermal effect and cavitation. The thermal effect results from the absorption of high energy by the targeted tissue and the conversion of that energy into heat, achieving temperatures between $65^{\circ} \mathrm{C}$ and $100^{\circ} \mathrm{C}$. Cavitation results from oscillation and subsequent violent collapse and energy dispersion of microbubbles in the targeted tissue because of their interaction with the ultrasonic energy (52). HIFU allows precise treatment of targeted areas without injury to surrounding soft tissue. Transrectal HIFU is used in the treatment of localized prostate cancer with MRI or ultrasound guidance (Figs. 2D-2H). In a study of 1,002 patients who underwent whole-gland ablation of localized prostate cancer, Crouzet et al. (53) found that the 8-y biochemical-free survival rates were $76 \%, 63 \%$, and $57 \%$, respectively, for low-, intermediate-, and high-risk patients. The prostate cancer-specific survival rate

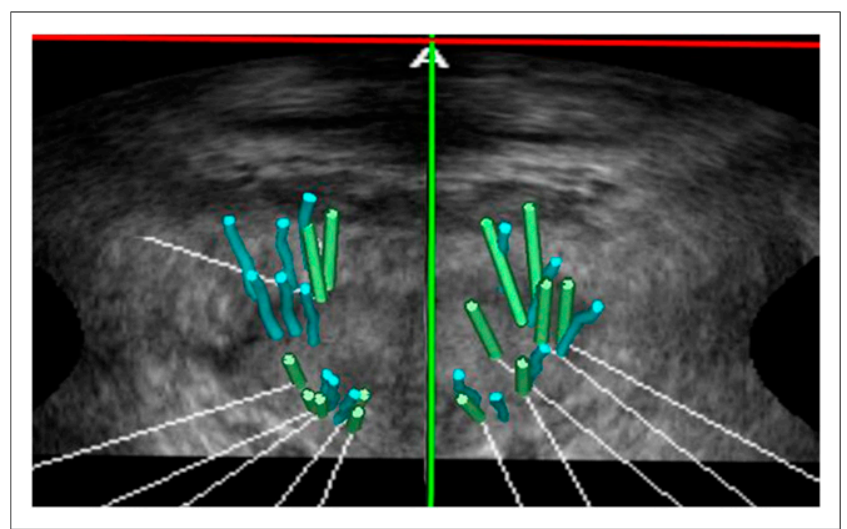

FIGURE 4. Novel TRUS technique for active surveillance (56). Single image from third-look follow-up 3-dimensional TRUS biopsy for active surveillance demonstrates 3-dimensional mapping biopsy trajectories using Urostation (Koelis) system. Blue bars represent location of cores from first biopsy. Green bars represent location of cores from current biopsy. Before firing actual TRUS-guided biopsy needle, virtual 3dimensional-simulated biopsy trajectory is created and superimposed on fusion image to confirm trajectory accuracy. Spatially trajectoryrecorded prostate biopsies allow accurate localization of areas of prior prostate cancer. 
and metastasis-free survival rate at $10 \mathrm{y}$ were $97 \%$ and $94 \%$, respectively. In a 14-y study of 538 patients, Ganzer et al. (54) found that the 5- and 10-y biochemical-free survival rates in patients who underwent HIFU treatment were $81 \%$ and $61 \%$, respectively, and concluded that HIFU may be a safe and effective treatment for localized prostate cancer in patients of advanced age and low-to-moderate risk and having a life expectancy of about $10 \mathrm{y}$. Adverse effects included bladder outlet obstruction $(28.3 \%)$; grades I, II, and III stress urinary incontinence $(13.8 \%, 2.4 \%$, and $0.7 \%$, respectively); and rectourethral fistula $(0.7 \%)$. In a more recent study of 918 patients, Uchida et al. (55) found that the 10 -y overall and cancerspecific survival rates of patients who underwent HIFU treatment of localized prostate cancer were $89.6 \%$ and $97.4 \%$, respectively. Adverse effects of HIFU treatment included urethral stricture (19.7\%), epididymitis $(6.2 \%)$, urinary incontinence $(2.3 \%)$, and rectourethral fistula $(0.1 \%)$.

The data on cryoablation and HIFU in the treatment of clinically localized prostate cancer are promising. More comprehensive research is currently under way to better characterize cryoablation and HIFU as options for the treatment of prostate cancer.

\section{CONCLUSION}

Ultrasound technology has undergone remarkable advances in recent years in terms of prostate cancer detection, biopsy, and localized treatment. In terms of prostate cancer detection, both CEUS and ultrasound elastography have better diagnostic accuracy than traditional TRUS, CDUS, and PDUS. In terms of prostate biopsy, although cancer detection rates for these procedures have not been shown to be sufficient to preclude systematic biopsy, novel ultrasound-guided techniques have been shown to improve the detection rate of prostate cancer when combined with systematic biopsy, while decreasing the number of biopsies required. In terms of localized treatment, both cryoablation and HIFU have shown promise in the treatment of localized prostate cancer. However, more studies on CEUS and ultrasound elastography must be performed, especially comparing them to multiparametric MRI in the detection and targeted biopsy of prostate cancer. Additional studies evaluating the clinical utility and treatment morbidity of cryoablation and HIFU are ongoing to better characterize the role of these novel ultrasound techniques in the treatment of prostate cancer.

\section{DISCLOSURE}

No potential conflict of interest relevant to this article was reported.

\section{REFERENCES}

1. Siegel RL, Miller KD, Jemal A. Cancer statistics, 2016. CA Cancer J Clin. 2016;66:7-30

2. Hankey BF, Feuer EJ, Clegg LX, et al. Cancer surveillance series: interpreting trends in prostate cancer-part I: evidence of the effects of screening in recent prostate cancer incidence, mortality, and survival rates. J Natl Cancer Inst. 1999;91:1017-1024.

3. Delongchamps NB, Singh A, Haas GP. The role of prevalence in the diagnosis of prostate cancer. Cancer Control. 2006;13:158-168.

4. Platz EA, Rimm EB, Willett WC, Kantoff PW, Giovannucci E. Racial variation in prostate cancer incidence and in hormonal system markers among male health professionals. J Natl Cancer Inst. 2000;92:2009-2017.

5. Loeb S, Bjurlin MA, Nicholson J, et al. Overdiagnosis and overtreatment of prostate cancer. Eur Urol. 2014;65:1046-1055.

6. Bjurlin MA, Carter HB, Schellhammer P, et al. Optimization of initial prostate biopsy in clinical practice: sampling, labeling, and specimen processing. $J$ Urol. 2013;189:2039-2046.
7. Scattoni V, Zlotta A, Montironi R, et al. Extended and saturation prostatic biopsy in the diagnosis and characterization of prostate cancer: a critical analysis of the literature. Eur Urol. 2007;52:1309-1322.

8. Ganie FA, Wanie MS, Ganie SA, et al. Correlation of transrectal ultrasonographic findings with histopathology in prostatic cancer [abstract]. J Educ Health Promot. 2014;3:38.

9. Engelbrecht MR, Barentsz JO, Jager GJ, et al. Prostate cancer staging using imaging. BJU Int. 2000;86:123-134.

10. Halpern EJ, Strup SE. Using gray-scale and color and power Doppler sonography to detect prostatic cancer. AJR. 2000;174:623-627.

11. Hricak H, Choyke PL, Eberhardt SC, Leibel SA, Scardino PT. Imaging prostate cancer: a multidisciplinary perspective. Radiology. 2007;243:28-53.

12. Ukimura O, Troncoso P, Ramirez El, Babaian RJ. Prostate cancer staging: correlation between ultrasound determined tumor contact length and pathologically confirmed extraprostatic extension. J Urol. 1998;159:1251-1259.

13. Rifkin MD, Sudakoff GS, Alexander AA. Prostate: techniques, results, and potential applications of color Doppler US scanning. Radiology. 1993;186:509-513.

14. Shigeno K, Igawa M, Shiina H, Wada H, Yoneda T. The role of colour Doppler ultrasonography in detecting prostate cancer. BJU Int. 2000;86:229-233.

15. Halpern EJ, Frauscher F, Strup SE, et al. Prostate: high-frequency Doppler US imaging for cancer detection. Radiology. 2002;225:71-77.

16. Kuligowska E, Barish MA, Fenlon HM, Blake M. Predictors of prostate carcinoma: accuracy of gray-scale and color Doppler US and serum markers. Radiology. 2001;220:757-764.

17. Cornud F, Hamida K, Flam T, et al. Endorectal color Doppler sonography and endorectal MR imaging features of nonpalpable prostate cancer: correlation with radical prostatectomy findings. AJR. 2000;175:1161-1168.

18. Sauvain JL, Sauvain E, Rohmer P, et al. Value of transrectal power Doppler sonography in the detection of low-risk prostate cancers. Diagn Interv Imaging. 2013;94:60-67.

19. Leen E, Averkiou M, Arditi M, et al. Dynamic contrast enhanced ultrasound assessment of the vascular effects of novel therapeutics in early stage trials. Eur Radiol. 2012;22:1442-1450.

20. Wilson SR, Greenbaum LD, Goldberg BB. Contrast-enhanced ultrasound: what is the evidence and what are the obstacles? AJR. 2009;193:55-60.

21. van Moorselaar RJ, Voest EE. Angiogenesis in prostate cancer: its role in disease progression and possible therapeutic approaches. Mol Cell Endocrinol. 2002; 197:239-250.

22. Brawer MK. Quantitative microvessel density: a staging and prognostic marker for human prostatic carcinoma. Cancer. 1996;78:345-349.

23. Weidner N, Carroll PR, Flax J, Blumenfeld W, Folkman J. Tumor angiogenesis correlates with metastasis in invasive prostate carcinoma. Am J Pathol. 1993; 143:401-409.

24. Sedelaar JP, van Leenders GJ, Hulsbergen-van de Kaa CA, et al. Microvessel density: correlation between contrast ultrasonography and histology of prostate cancer. Eur Urol. 2001;40:285-293.

25. Sano F, Uemura H. The utility and limitations of contrast-enhanced ultrasound for the diagnosis and treatment of prostate cancer. Sensors (Basel). 2015; 15:4947-4957.

26. Matsumoto K, Nakagawa K, Hashiguchi A, et al. Contrast-enhanced ultrasonography of the prostate with Sonazoid. Jpn J Clin Oncol. 2010;40:1099-1104.

27. Strazdina A, Krumina G, Sperga M. The value and limitations of contrastenhanced ultrasound in detection of prostate cancer. Anticancer Res. 2011;31: 1421-1426.

28. Li H, Xia J, Xie S, et al. Prostate cancer: a comparison of the diagnostic performance of transrectal ultrasound versus contrast enhanced transrectal ultrasound in different clinical characteristics. Int J Clin Exp Med. 2015;8:2142821434.

29. Li Y, Tang J, Fei X, Yi G. Diagnostic performance of contrast enhanced ultrasound in patients with prostate cancer: a meta-analysis. Acad Radiol. 2013; 20:156-164.

30. Harvey CJ, Pilcher J, Richenberg J, Patel U, Frauscher F. Applications of transrectal ultrasound in prostate cancer. Br J Radiol. 2012;85(suppl):S3-S17.

31. Goossen TE, de la Rosette JJ, Hulsbergen-van de Kaa CA, van Leenders GJ, Wijkstra H. The value of dynamic contrast enhanced power Doppler ultrasound imaging in the localization of prostate cancer. Eur Urol. 2003;43:124-131.

32. Zhu Y, Chen Y, Jiang J, et al. Contrast-enhanced harmonic ultrasonography for the assessment of prostate cancer aggressiveness: a preliminary study. Korean $J$ Radiol. 2010;11:75-83.

33. Good DW, Stewart GD, Hammer S, et al. Elasticity as a biomarker for prostate cancer: a systematic review. BJU Int. 2014;113:523-534.

34. Tsutsumi M, Miyagawa T, Matsumura T, et al. Real-time balloon inflation elastography for prostate cancer detection and initial evaluation of clinicopathologic analysis. AJR. 2010;194:W471-W476. 
35. Aboumarzouk OM, Ogston S, Huang Z, et al. Diagnostic accuracy of transrectal elastosonography imaging for the diagnosis of prostate cancer: a systematic review and meta-analysis. BJU Int. 2012;110:1414-1423.

36. Zhang B, Ma X, Zhan W, et al. Real-time elastography in the diagnosis of patients suspected of having prostate cancer: a meta-analysis. Ultrasound Med Biol. 2014;40:1400-1407.

37. Junker D, Schafer G, Kobel C, et al. Comparison of real-time elastography and multiparametric MRI for prostate cancer detection: a whole-mount step-section analysis. AJR. 2014;202:W263-W269.

38. Sarkar S, Das S. A review of imaging methods for prostate cancer detection. Biomed Eng Comput Biol. 2016;7:1-15.

39. Postema A, Mischi M, de la Rosette J, Wijkstra H. Multiparametric ultrasound in the detection of prostate cancer: a systematic review. World J Urol. 2015;33: 1651-1659.

40. Correas JM, Tissier AM, Khairoune A, et al. Ultrasound elastography of the prostate: state of the art. Diagn Interv Imaging. 2013;94:551-560.

41. Boehm K, Salomon G, Beyer B, et al. Shear wave elastography for localization of prostate cancer lesions and assessment of elasticity thresholds: implications for targeted biopsies and active surveillance protocols. J Urol. 2015;193:794800 .

42. Ahmad S, Cao R, Varghese T, Bidaut L, Nabi G. Transrectal quantitative shear wave elastography in the detection and characterisation of prostate cancer. Surg Endosc. 2013;27:3280-3287.

43. Porsch M, Wendler JJ, Liehr UB, et al. New aspects in shear-wave elastography of prostate cancer. J Ultrason. 2015;15:5-14.

44. Hodge KK, McNeal JE, Stamey TA. Ultrasound guided transrectal core biopsies of the palpably abnormal prostate. J Urol. 1989;142:66-70.

45. Taneja SS, Bjurlin MA, Carter HB, et al. White paper: AUA/optimal techniques of prostate biopsy and specimen handling. American Urological Association website. https://www.auanet.org/common/pdf/education/clinical-guidance/ Prostate-Biopsy-WhitePaper-Updated2015.pdf. Published 2013. Updated 2015. Accessed August 15, 2016.
46. Bjurlin MA, Wysock JS, Taneja SS. Optimization of prostate biopsy: review of technique and complications. Urol Clin North Am. 2014;41:299-313.

47. van Hove A, Savoie PH, Maurin C, et al. Comparison of image-guided targeted biopsies versus systematic randomized biopsies in the detection of prostate cancer: a systematic review of well-designed studies. World J Urol. 2014;32:847858 .

48. de Rooij M, Hamoen EH, Futterer JJ, Barentsz JO, Rovers MM. Accuracy of multiparametric MRI for prostate cancer detection: a meta-analysis. AJR. 2014;202:343-351.

49. Valerio M, Donaldson I, Emberton M, et al. Detection of clinically significant prostate cancer using magnetic resonance imaging-ultrasound fusion targeted biopsy: a systematic review. Eur Urol. 2015;68:8-19.

50. Onik GM, Cohen JK, Reyes GD, et al. Transrectal ultrasound-guided percutaneous radical cryosurgical ablation of the prostate. Cancer. 1993;72:1291-1299.

51. Marien A, Gill I, Ukimura O, Betrouni N, Villers A. Target ablation: imageguided therapy in prostate cancer. Urol Oncol. 2014;32:912-923.

52. Halpern EJ. Science to practice: high-intensity focused ultrasound ablation-will image-guided therapy replace conventional surgery? Radiology. 2005;235:345346.

53. Crouzet S, Chapelon JY, Rouviere O, et al. Whole-gland ablation of localized prostate cancer with high-intensity focused ultrasound: oncologic outcomes and morbidity in 1002 patients. Eur Urol. 2014;65:907-914.

54. Ganzer R, Fritsche HM, Brandtner A, et al. Fourteen-year oncological and functional outcomes of high-intensity focused ultrasound in localized prostate cancer. BJU Int. 2013;112:322-329.

55. Uchida T, Tomonaga $\mathrm{T}$, Kim H, et al. Improved outcomes with advancements in high intensity focused ultrasound devices for the treatment of localized prostate cancer. J Urol. 2015;193:103-110.

56. Ukimura O, Gross ME, de Castro Abreu AL, et al. A novel technique using three-dimensionally documented biopsy mapping allows precise re-visiting of prostate cancer foci with serial surveillance of cell cycle progression gene panel. Prostate. 2015;75:863-871. 